\title{
The observation of lightning-related events with the Surface Detector of the Pierre Auger Observatory
}

\author{
Roberta Colalillo ${ }^{1, *}$ for the Pierre Auger Collaboration ${ }^{2, * *, * * *}$ \\ ${ }^{1}$ INFN, Sezione di Napoli, Italy \\ ${ }^{2}$ Observatorio Pierre Auger, Av. San Martin Norte 304, 5613 Malargüe, Argentina
}

\begin{abstract}
The Pierre Auger Observatory, designed to detect ultra-high energy cosmic rays, can be a valid instrument at the ground to study phenomena related to the atmospheric electricity. The fluorescence detector is a powerful instrument to observe ELVES thanks to its excellent time resolution, while peculiar events with a large number of triggered stations have been recorded by the surface detector. The characteristic signal of these events lasts more than $10 \mu \mathrm{s}$, about two orders of magnitude more than the duration of a signal produced by a cosmic muon. Moreover, each of these events has at least one station with a signal dominated by a high-frequency noise that could be related with a lightning-induced signal. Stations with a long-lasting signal are arranged in a disk shape. There are "big" events characterized by a radius of about $6 \mathrm{~km}$ and few "small" events with a radius of about $2-3 \mathrm{~km}$. The signal, generated by a source very close to the ground, first reaches the innermost stations and then spreads outwards. In the "big" events, a lack of signal in some of the central stations was observed. Further studies and checks are in progress to understand the origin of the lack of signal and what mechanisms occurring during the lightning evolution may provide for electric fields capable of generating and accelerating particles that can produce Cherenkov light in the stations of the surface detector.
\end{abstract}

\section{Introduction}

The Pierre Auger Observatory [1] is the largest facility in the world to study ultra-high energy cosmic rays. It is located on a high plain in western Argentina, whose mean altitude is about $1400 \mathrm{~m}$ a.s.l., and cover an area of about $3000 \mathrm{~km}^{2}$. Its main characteristic is the hybrid detection technique based on the combined use of two different instruments: the Fluorescence Detector (FD) and the Surface Detector (SD). The longitudinal profile of a cosmicray shower can be reconstructed thanks to the fluorescence light $(300-400 \mathrm{~nm})$ produced by the interaction of the shower particles with the Earth's atmosphere and collected at the four sites of the FD. Light in the same waveband is produced at the base of the ionosphere when an electromagnetic pulse is emitted by lightning developed more than $600 \mathrm{~km}$ from the observatory and not visible from the site. The FD has turned out to be a valid instrument for studying this transient light, the ELVES light [2]. Also, the SD, composed of 1660 water-Cherenkov Detectors (WCDs) arranged in a triangular grid with nearest neighbors separated by $1.5 \mathrm{~km}$ and designed to study the distribution of shower particles at the ground, is involved in atmospheric electricity studies. Among the events collected by the SD, some exotic events with a radially expanding footprint and correlated with lightning strikes were found [3]. Many SD stations are triggered by these events. Each

\footnotetext{
*e-mail: colalillo@na.infn.it

**e-mail: auger_spokespersons@fnal.gov

***Authors list: http://www.auger.org/archive/authors_2018_09.html
}

WCD consists of $3.6 \mathrm{~m}$ diameter tank containing a liner with a reflective inner surface, which is filled with 12,000 liters of ultra-pure water. When a charged particle with a speed higher than that of light in water crosses the detector, Cherenkov light is produced, and then collected by three PMTs. Each PMT has two readout channels, the High Gain (HG) channel that is taken from the anode, and the low gain channel from the last dynode. There is an amplification factor of about 32 between the two channels. In many cases, the HG channel is saturated by the exotic event signal, and it is possible to reproduce the light curve only thanks to the LG channel. The two output signals are processed by six FADCs with a sampling rate of $40 \mathrm{MHz}$. The SD acquisition window, optimized for cosmic-ray signals, lasts $19.2 \mu \mathrm{s}$.

The Observatory makes use of the atmosphere as a giant calorimeter. For this reason, many facilities for the atmospheric monitoring are present on the site. In the last years, the Auger Engineering Radio Array (AERA) for the detection of cosmic-ray radio emission, that is influenced by lightning activity, was completed and the Observatory further widened its research horizons to atmospheric electricity studies. Therefore, a new lightning detection system was installed [4]. Five Boltek StormTrackers that are able to detect lightning strikes within a radius of $1000 \mathrm{~km}$ were added to the monitoring devices together with an E-field mill at the AERA site. A schematic overview of the Observatory with all atmospheric monitoring facilities is shown in Figure 1. 


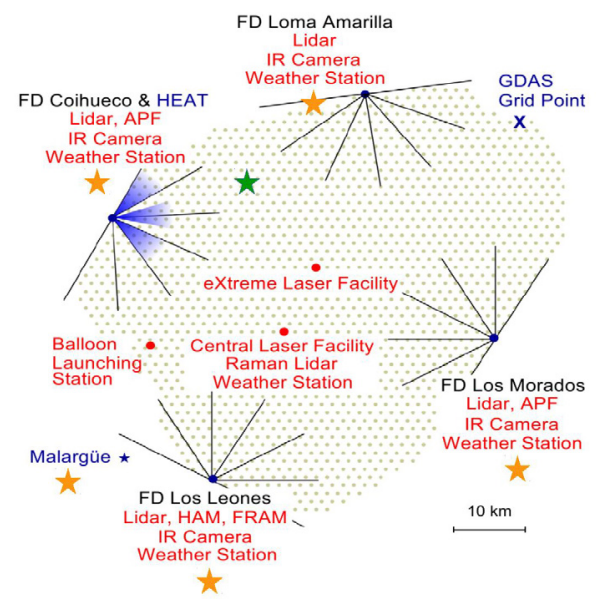

Figure 1. Schematic overview of the Pierre Auger Observatory. The beige dots represent the SD stations, the black lines the field of view of the FD telescopes. The Boltek StormTrackers are indicated by the orange stars, while the E-field mill is indicated by the green one. Also the other atmospheric monitoring devices are shown.

\section{SD Exotic Events}

The exotic events observed with the SD have three main characteristics: many triggered detectors arranged in a disk shape; signals which are about two orders of magnitudes longer than signals produced by cosmic muons; stations with a signal dominated by high-frequency noise, called "lightning stations". The presence of these last stations suggests that exotic events could have been produced during thunderstorms. Finally, in these events, there are also stations, called "muon stations", with a signal produced by a cosmic muon, but they are not correlated in time, and, in many cases, neither in space with the "longsignal stations". A long-lasting signal, a "lightning station" and a typical footprint of these exotic events are represented in Figure 2. Before starting the characterization and reconstruction of these very peculiar events, it is important to check that they have a physical origin, and are not an artifact due to electromagnetic noise. If the longlasting signals are produced by the passage of charged particles through the water, we expect to register the same signal with the three PMTs and with the two readout channels of each PMT. We calculated the ratio between the signals of the two PMTs at a time bin by bin for each station and then an average ratio. The distribution of these ratios is peaked at 1 as expected. In a similar way, for each PMT, we verified the consistency between the HG signal and the LG signal multiplied by the amplification factor obtaining again a distribution peaked at 1 .

The data sample used for this analysis consists of all SD events collected since January 2004 up to 15 May 2017 which pass the first three SD trigger levels ( $\sim 10^{7}$ events). The third level trigger is the first global trigger necessary to define the event [5]. The first requirement for these events is the presence of at least a lightning station. In this way, we selected about 200000 events. In this sample, we searched for those events with at least 10 long- signal stations. A station is accepted if at least the signal of two PMTs fulfill the long-signal condition: on a smeared HG trace, at least 80 consecutive bins above 1 baseline error are requested. With this search algorithm, 28 events were identified; among them, 16 events, the "large events", have more than 20 long-lasting stations, arranged in a ring shape with a radius that spans from 4 to $8 \mathrm{~km}$.

\section{The Long-Lasting Signal}

The long-lasting signals of our exotic events are not usually fully contained in our acquisition time window. To recover the missing part, we need to identify a function that describes the shape of our signal. Studying a small sample of completely acquired signals, we found that the asymmetric Gaussian distribution best describes the leading and falling edge of the signal. A chi-square minimization is performed to fit the signal. An example of the fit is shown in Figure 3. We require that the Gaussian peak is between $2.5 \mu \mathrm{s}$ and $16.7 \mu \mathrm{s}$ of the DAQ window to see at least a small part of the leading and falling edge and estimate in a proper way all the four parameters of the asymmetric Gaussian. We accept the fit if the percentage difference between the sum of the content of the trace bins and the integral of the fitting function in our time window is less than $5 \%$, and if the duration of the total fitting function is less than $100 \mu \mathrm{s}$. The station is tagged as "good station" if the fitting procedure was successful for at least two PMTs. Studying the parameters of the "good fits", some general features of the signal were identified: the rise time of the signal $\left(r_{i} \sigma_{i}\right)$ is smaller than the fall time $\left(\sigma_{i}\right), \sigma_{i}$ is bigger than $2.5 \mu \mathrm{s}$, and $r_{i} \sigma_{i}$ is constant as a function of $\sigma_{i}^{2}$. The signal detected by each PMT is given by the total integral of the fitting function. The signal per station is the mean of the three PMT signals.

The start time of our long-lasting signals is defined as the time corresponding to the $10 \%$ of the fit function's peak value, $t_{10}$ (see Figure 3 ). This time plus the GPS time of the event defines the arrival time of the signal in the station.

\section{Event Development}

The first step to characterize the exotic events and their evolution is the reconstruction of their footprint at the ground. We performed an unweighted chi-square minimization on the station coordinates to find the center of the disk and its radius. We identified two categories of events: the "large" and "small" events. An example per category is shown in Figure 4. The large events are characterized by more than 20 triggered stations with a longlasting signal, that are arranged in a ring shape. The origin of the depletion of the signal at the center of the footprint is being investigated. It could be physical or related to the Auger trigger, that is optimized for the cosmic-ray detection. There are many differences between signals produced by cosmic rays and by our exotic events, but in this case, first of all, we have to consider that the number of stations triggered by exotic events is much bigger. Moreover, as we will see later, also the possible presence of 

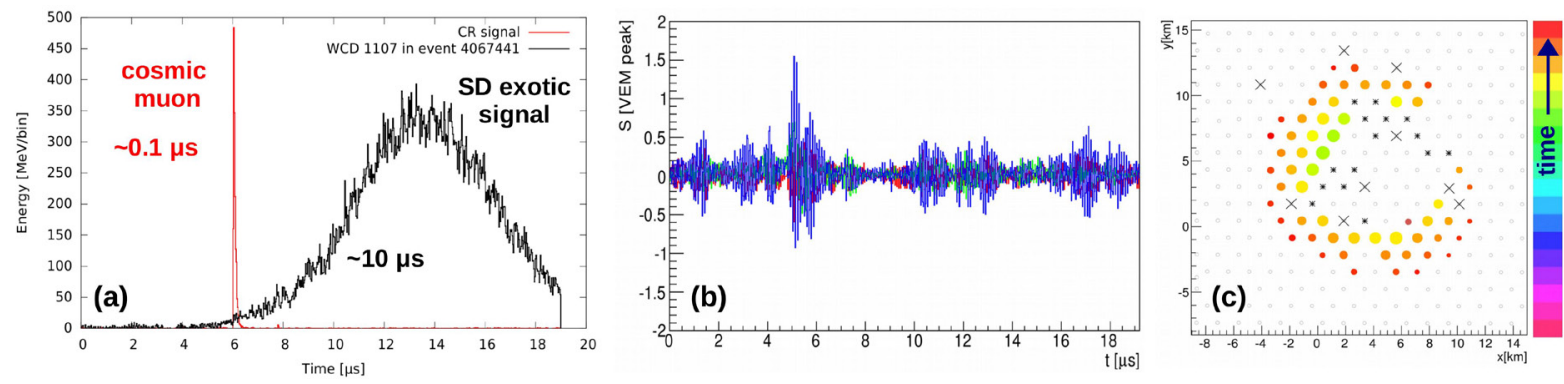

Figure 2. (a) A typical signal of the exotic events compared with a signal from a cosmic muon. (b) A lightning signal in a station: the three colors represent the signal collected by the three PMTs. (c) A typical footprint of an exotic event. The colored circles represent the long-signal stations. The marker size is related to the intensity of the signal, the color to the arrival time. The asterisks represent the lightning stations, and the crosses the muon stations.

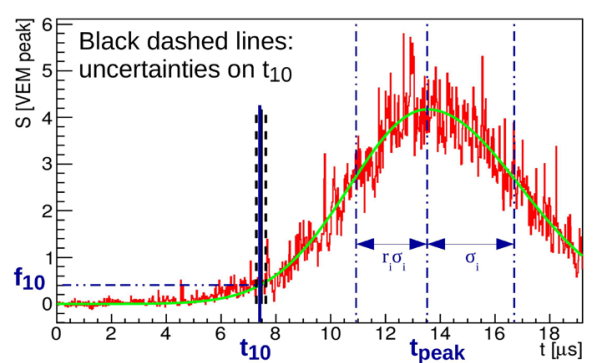

Figure 3. A typical long-lasting signal perfectly described by an asymmetric Gaussian distribution. The start time of the signal, indicated by the full blue line, is the time corresponding to the $10 \%$ of the fit function's peak value.
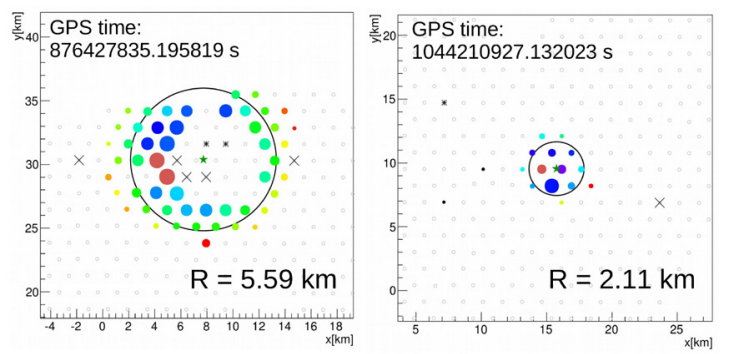

Figure 4. Footprint at the ground for a "large" (left) and "small" (right) event. The large event presents a ring footprint; the origin of the depletion of the signal at the center of the disk is not clear yet.

lightning strikes could modify the detection. The radius of large events spans from 4 to $8 \mathrm{~km}$. The small events, on the contrary, present a compact footprint and their radius is about $2-3 \mathrm{~km}$.

At this point, we can study how the signal changes as a function of the distance from the center of the footprint for the "good events", that are those events with at least eight "good stations". The signal is bigger close to the center and decreases going towards the external stations. In Figure 5, the logarithm of the signal as a function of the distance from the center is shown and it is well described by a parabola.

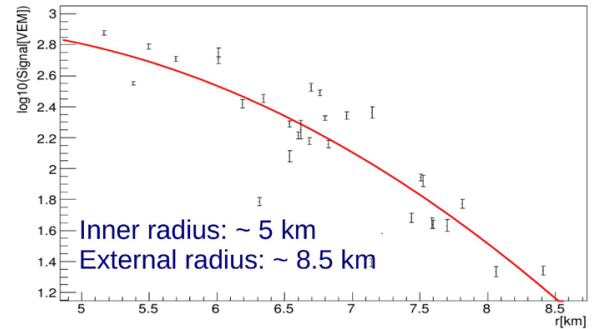

Figure 5. Logarithm of the long-lasting signal as a function of the distance from the center of the footprint described by a parabola.

We can easily transform the signal of each station in deposited energy considering that $1 \mathrm{MeV} \sim 240 \mathrm{VEM}$ (Vertical Equivalent Muon, the signal released by a muon crossing a WCD vertically at its center). The energy per longsignal station spans from $\sim 10^{4} \mathrm{MeV}$ to $\sim 10^{6} \mathrm{MeV}$. We can calculate the total energy deposited at the ground by an event multiplying the energy per $\mathrm{m}^{2}$ by the real area covered by the active stations. The obtained energies oscillate between $10^{17} \mathrm{eV}$ and $10^{18} \mathrm{eV}$. If the lack of the signal at the center of the footprint is not physical, we are underestimating this energy. With the same method, we can calculate the energy deposited at the ground by a cosmicray shower initiated by a proton with energy $10^{19} \mathrm{eV}$. That deposited energy is about two orders of magnitude lower than the energy released by an exotic event. Moreover, we can compare the energy per $\mathrm{m}^{2}$ of an exotic event with the energy per $\mathrm{m}^{2}$ measured by other experiments which detected radiation bursts. For example, Dwyer et al. work at about $100 \mathrm{~m}$ from the lightning strike with NaI scintillators [6]; their energy density is consistent with our energy density at $\sim 0.5 \mathrm{~km}$ from the center or farther. The TA experiment, instead, measured $\sim 10^{2} \mathrm{MeV}$ per $\mathrm{m}^{2}$ with plastic scintillators [7]. This energy density is two orders of magnitude lower than our smallest one, but in our evaluations we have not considered the photon detection efficiency, that is small for plastic scintillators.

Finally, we assumed that our events temporally develop according to a spherical propagation front and performed a three-dimensional fit with four free parameters: $x_{0}, y_{0}$, 


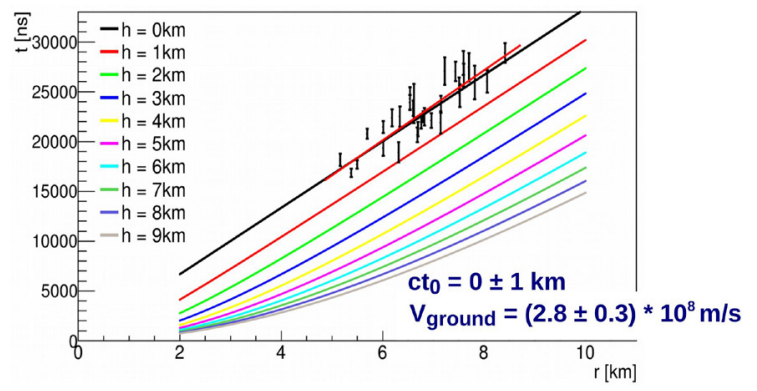

Figure 6. Arrival times as a function of the distance from the center. The black points are the measured arrival times of the long-signal stations with their uncertainty for one event. The colored lines represent the simulated arrival times at the ground obtained by a simple $\mathrm{MC}$ which assumes a spherical propagation front. Each line corresponds to a different altitude of the source point, from 0 to $10 \mathrm{~km}$. Data are compatible with a source very close to ground.

and $c t_{0}$, that are the three coordinates of the source point of the event, and $t_{O f f}$, which takes into account the offset between the starting time of the simulated event, $t_{0}$, and the GPS time of the real event. We obtained good fits where the altitude of the source never exceed $1 \mathrm{~km}$. Moreover, we compared our measured arrival times with the arrival times at the ground obtained by a simple geometrical Toy MC always based on a spherical propagation front. The colored lines shown in Figure 6 represent the arrival times otained changing the altitude of the source from 0 to 10 $\mathrm{km}$ in the simulation. Subtracting $t_{O f f}$ obtained with the three-dimensional fit from the measured arrival times, they match the line corresponding to an altitude of $0 \mathrm{~km}$. This result is compatible with the one obtained from the threedimensional fit: a source very close to the ground with a height below $1 \mathrm{~km}$. Fitting with a straight line $(t=a+b s$, with $b=\frac{1}{v}$ ) the measured arrival time as a function of the distance from the center of the footprint, we can obtain the propagation velocity at the ground. We found values close to the speed of light, as expected for events with a source at the ground. Knowing the internal and external radius of the footprint and the propagation velocity, we can calculate also the propagation time at the ground, that is of the same order of magnitude as the duration of the long-lasting signal. The small event shown on the right of Figure 4 does not pass all the quality cuts requested for a reliable reconstruction of the event because many long-lasting signals do not have the peak in the DAQ window. Anyway, performing a raw reconstruction, we obtained also for this event that the signal starts from the inner part of the footprint, where it is bigger, and moves toward the outer part at the speed of light. Moreover, the source is close to the ground.

To validate the hypothesis suggested by the presence of lightning stations that our exotic events happened during thundertorms, we compared the small sample of "good events", consisting of ten events, with the lightning strikes collected by WWLLN (World Wide Lightning Location Network), that has antennas also in South America. We found that $70 \%$ of the events in the sample are correlated in time within $1 \mathrm{~ms}$ with WWLLN lightning strikes. Also the spatial correlation is very good considering that the global location accuracy for WWLLN network assuming 5-station involvement ranges over 1.9 to $19 \mathrm{~km}$ [8].

\section{Conclusion}

Peculiar events related to lightning were observed with the surface detector of the Pierre Auger Observatory. These events are characterized by signals which last $\sim 10 \mu$ s, two orders of magnitude longer than signals produced by cosmic muons. The large events contain more than 20 longsignal stations that are arranged in a ring shape with an average radius of $6 \mathrm{~km}$. The signal of both the small and the large events expands radially at the speed of light and is bigger in the inner part of the disk and decreases with the increasing of the distance from the center. Moreover, the timing evolution of these events is compatible with a spherical front moving at the speed of light with a source very close to the ground. At this moment, we do not know which phenomenon can explain our observations. The attachment process [9], that is the meeting of the downward lightning stepped leader with an upward leader at a few tens of meters above the ground, could be a possible explanation of our events, as also TGEs (Thunderstorm Ground Enhancement) [10]. They originate from electron acceleration and multiplication processes in the strong electric fields in thunderclouds. Detailed studies of the environmental conditions in correspondence with our events and comparisons with simulations are necessary to understand the origin of our exotic events.

\section{Acknowledgments}

The authors wish to thank the World Wide Lightning Location Network (wwlln.net), a collaboration among over 50 universities and institutions, for providing the lightning location data used in this paper.

\section{References}

[1] Pierre Auger Coll., Nucl. Instrum. Meth. A 798, 172-213 (2015).

[2] R.Mussa et al., EPJ Plus 127, 94 (2012).

[3] R. Colalillo, for the Pierre Auger Coll., 35th Int. Cosmic Ray Conf., Busan, Korea (2017).

[4] J. Rautenberg, for the Pierre Auger Coll., 34th Int. Cosmic Ray Conf., The Hague (2015).

[5] Pierre Auger Coll., Nucl. Instrum. Meth. A 613, 29-39 (2010).

[6] J.R. Dwyer et al., Geophys. Res. Lett. 31, L05119 (2004).

[7] Telescope Array Coll., J. Geophys. Res. Atmos. 123 (2018).

[8] C.J. Rodger, J.B. Brundell, and R.L. Dowden, Ann. Geophys. 23, 277-290 (2005).

[9] J. Jerauld et al., J. Geophys. Res 112, D13113 (2007).

[10] A. Chilingarian et al., Phys. Rev. D 98, 082001 (2018). 